\title{
Concurrent scale-free and small-world networks support criticality in cortical ensembles
}

\author{
Paolo Massobrio ${ }^{1 *}$, Valentina Pasquale ${ }^{2}$, Sergio Martinoia ${ }^{1}$ \\ From Twenty Second Annual Computational Neuroscience Meeting: CNS*2013 \\ Paris, France. 13-18 July 2013
}

Nowadays, it is widely accepted that structural features of cortical networks are tightly linked to aspects of brain function, playing a crucial role in determining which electrophysiological patterns (and thus, brain states) can and cannot occur. In this work, we investigated the interplay between network topology and spontaneous dynamics within the framework of neuronal avalanches and self-organized criticality [1].

The aim of this study is to sustain the hypothesis that the emergence of critical states, which in their turn would optimize functional properties in the cortex, is supported by specific complex network topologies.

We followed a computational approach by developing large-scale network models mimicking the electrophysiological patterns (i.e., spiking and bursting activity) displayed by in vitro cortical networks coupled to MicroElectrode Arrays (MEAs). In this preparation, neurons are able to freely re-create networks that exhibit complex and highly variable spatio-temporal patterns of activity [2]. As neurons grow without external constraints and self-organize depending on many parameters, we do not have any clue about the resulting morphological connectivity. Moreover, some cultures actually exhibit scale-free distributions of neuronal avalanches, a hallmark of SOC, thus demonstrating that they preserve self-organization properties featured by in vivo-formed cell assemblies [3]. Therefore, cortical networks actually show different states (critical, subcritical or supercritical), but their relationship with the underlying connectivity remains unknown [4]. Due to the difficulties of determining the network topology of our cultures from a limited number of recording sites (60 microelectrodes), we took advantage of a computational model consisting of a neuronal

\footnotetext{
* Correspondence: paolo.massobrio@unige.it

'Department of Informatics, Bioengineering, Robotics and Systems

Engineering (DIBRIS), University of Genova, Genova, 16145, Italy

Full list of author information is available at the end of the article
}

network made up of 1024 Izhikevich neurons [5]. Network topologies were designed following the canonical architectures scale-free, random, and small-world [6]. We simulated the spontaneous activity of such neuronal networks, by sweeping the most common parameters used to characterize these graphs, such as clustering coefficient, connection density, etc. [7]. The main finding which emerges is that although all the network configurations determine a mix of spiking, and bursting activity, the scale-free with small-world features display a critical behavior.

\section{Acknowledgements}

The research leading to these results has received funding from the European Union's Seventh Framework Programme (ICT-FET FP7/2007-2013, FET Young Explorers scheme) under grant agreement n² 284772 (BrainBow).

\section{Author details}

'Department of Informatics, Bioengineering, Robotics and Systems Engineering (DIBRIS), University of Genova, Genova, 16145, Italy. ${ }^{2}$ Department of Neuroscience and Brain Technologies - NTECH, Istituto Italiano di Tecnologia (IIT), Via Morego 30, 16163, Genova, Italy.

Published: 8 July 2013

\section{References}

1. Beggs JM, Plenz D: Neuronal avalanches in neocortical circuits. J Neurosci 2003, 23:11167-11177.

2. Wagenaar DA, Pine J, Potter SM: An extremely rich repertoire of bursting patterns during the development of cortical cultures. BMC Neurosci 2006, 7.

3. Petermann T, Thiagarajan TC, Lebedev MA, Nicolelis MA, Chialvo DR, Plenz D: Spontaneous cortical activity in awake monkeys composed of neuronal avalanches. PNAS 2009, 106:15921-15926.

4. Pasquale V, Massobrio P, Bologna LL, Chiappalone M, Martinoia S: Selforganization and neuronal avalanches in networks of dissociated cortical neurons. Neuroscience 2008, 153:1354-1369.

5. Izhikevich EM: Simple model of spiking neurons. IEEE Trans Neur Net 2003, 6:1569-1572.

6. Albert R, Barabasi A-L: Statistical mechanics of complex networks. Rev Mod Phys 2002, 74:47-97.

7. Bullmore E, Sporns O: Complex brain networks: graph theoretical analysis of structural and functional systems. Nat Rev 2009, 10:186-198.

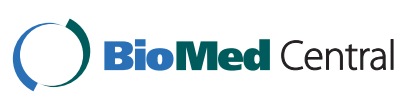


doi:10.1186/1471-2202-14-S1-P264

Cite this article as: Massobrio et al:: Concurrent scale-free and smallworld networks support criticality in cortical ensembles. BMC Neuroscience 2013 14(Suppl 1):P264.

Submit your next manuscript to BioMed Central and take full advantage of:

- Convenient online submission

- Thorough peer review

- No space constraints or color figure charges

- Immediate publication on acceptance

- Inclusion in PubMed, CAS, Scopus and Google Scholar

- Research which is freely available for redistribution 